www.nature.com/ejhg

\title{
Parametric and non-parametric linkage analysis of several candidate regions for genes for human handedness
}

\author{
Tom Van Agtmael ${ }^{*, 1,2,3}$, Susan M Forrest ${ }^{1,2}$ and Robert Williamson ${ }^{1,2}$ \\ ${ }^{1}$ Murdoch Childrens Research Institute, Royal Children's Hospital, Parkville, Melbourne, Australia; ${ }^{2}$ Department of \\ Paediatrics, Royal Children's Hospital, The University of Melbourne, Melbourne, Australia
}

The frequency of left-handedness in the general population is around $11 \%$. Both environmental and genetic models have been proposed to explain the aetiology of human handedness. The majority of genetic models, such as those of Annett, McManus and Klar, propose a single gene determinant with a non-Mendelian inheritance pattern. As left-handedness is correlated with cerebral asymmetry and is a feature of left-right asymmetry, genes involved in the development of left-right asymmetry can be considered as candidate genes. Candidate gene analysis was performed using an informative extended pedigree, and also using nuclear families of right-handed parents with left-handed children. Segregation analysis in the extended pedigree identified allele sharing in the NODAL and DNAHC13 candidate regions on chromosome 10 and 1. Linkage analysis using the models of Klar and McManus, and non-parametric analysis on nuclear families, subsequently excluded all candidate regions tested. This demonstrates the power to identify the genes specifying handedness by the conduct of extended genetic studies on these and similar cohorts.

European Journal of Human Genetics (2002) 10, 623-630. doi:10.1038/sj.ejhg.5200851

Keywords: left-handedness; linkage analysis; handedness

\section{Introduction}

Left-handedness has a frequency of $10-12 \%$ in the general population with a higher frequency of left-handedness in the male population compared to the female population. ${ }^{1}$ Left-handedness has been correlated with a number of other traits ranging from risk of breast cancer, ${ }^{2}$ auto immune disorders, decreased survival fitness and a better recovery from stroke. ${ }^{3}$

A relationship exists between cerebral dominance and handedness. Left-hemisphere dominance occurs in $97 \%$ of right-handed people, but only in $70 \%$ of left-handed people. The other $30 \%$ of left-handed individuals have anomalous or right-hemisphere dominance. ${ }^{4,5}$

\footnotetext{
${ }^{*}$ Correspondence: Tom Van Agtmael, Human Genetics Unit, Western General Hospital, Crewe Road, Edinburgh EH2 4XU, UK.

Tel +44 (0)131332 2471; Fax: +44 (0)131343 2620;

E-mail: tom.vanagtmael@hgu.mrc.ac.uk

${ }^{3}$ Current address: MRC Human Genetics Unit, Edinburgh, UK

Received 14 December 2001; revised 6 June 2002; accepted 7 June 2002
}

The genetics of handedness in humans is controversial. Both environmental and genetic models have been proposed to explain the genetics of human handedness. Several genetic models have been suggested that allow for the influence of other, yet to be determined, factors by using the biological mechanism of fluctuating asymmetry. Fluctuating asymmetry postulates that handedness is determined by chance due to noise and stochastic events during development. ${ }^{6}$ Hence fluctuating asymmetry will result in 50\% left-handedness and 50\% right-handedness. ${ }^{6}$

McManus proposed a genetic model where a single gene, having two alleles $\mathrm{C}$ and $\mathrm{D}$, is responsible for lefthandedness. ${ }^{6}$ Individuals with the homozygous D/D genotype will be right-handed, while heterozygotes will have a $75 \%$ chance of becoming right-handed. The homozygous C/C genotype will exhibit fluctuating asymmetry. ${ }^{6} \mathrm{Klar}^{7}$ proposed a single gene model similar to the one of McManus. A gene, RGHT, has two alleles $\mathrm{R}$ and $\mathrm{r}$ for 
which genotypes $\mathrm{R} / \mathrm{R}$ and $\mathrm{R} / \mathrm{r}$ will result in right-handedness and genotype $\mathrm{r} / \mathrm{r}$ in fluctuating asymmetry. ${ }^{7}$ Klar calculated the gene frequencies for $\mathrm{R}$ and $\mathrm{r}$ to be 58 and $42 \%$ respectively. ${ }^{7}$

Due to the relation between left-handedness and cerebral asymmetry, we hypothesised that genes involved in the development of left-right asymmetry can be considered as candidate genes. The gene Lrd, located on mouse chromosome 12 , causes murine situs inversus and codes for a dynein heavy-chain molecule. ${ }^{8}$ The localisation of other murine heavy chain dyneins has been determined by Vaughan et al. ${ }^{9}$ Because a dynein molecule is involved in the development of left-right asymmetry, and is thus a candidate gene for handedness, other dyneins can also be considered as candidate genes. Another gene that plays a crucial role in the development of left-right asymmetry is Nodal. ${ }^{10}$ Nodal is a member of the TGFb superfamily of secreted proteins and is one of the key players in the L-R asymmetry pathway. Nodal is asymmetrically expressed in the node and in the left lateral plate mesoderm in the developing mouse embryo. ${ }^{10}$

In this study we report the analysis of several candidate regions for left-handedness in an extended family and several nuclear families. Both parametric and non-parametric analyses were performed which permitted exclusion of several candidate regions harbouring genes with the models of Klar or McManus.

\section{Materials and methods}

\section{Sample collection}

The study was approved by the human research ethics committee of the Women and Children's Hospital, Melbourne, Australia. We collected samples of one large extended pedigree and 27 nuclear families consisting of two right-handed parents and at least two left-handed children. Nuclear families consisting of right-handed parents with left-handed children are the most informative pedigree structure for linkage calculations using the model of Klar, as it maximises the proportion of parents that are heterozygous $\mathrm{R} / \mathrm{r}$ at the locus level. ${ }^{11}$ Consequently, and analogous to standard autosomal recessive disorders, the heterozygosity of the right-handed parents and the homozygosity of the left-handed children at the locus level, will result in informative meioses and more power in linkage calculations. $^{11}$

All families are of Caucasian origin and living in Australia. We collected a total of 173 individuals, 107 of who were right-handed $(62 \%)$ and 66 of who were lefthanded (38\%). Handedness was assessed using the Edinburgh Inventory, ${ }^{12}$ which was posted to participants. Laterality quotient scores (LQ scores) were calculated for every participant. ${ }^{12}$ Individuals were excluded from the study if their LQ scores and handedness for writing were contradictory.

\section{Sample preparation}

Mouthwashes were used as the primary source of DNA. ${ }^{13}$ The buccal cells in the $10 \mathrm{ml}$ water mouthwash were pelleted down by centrifugation for $15 \mathrm{~min}$ at $3000 \mathrm{rpm}$. Following resuspension of the pellet in $300 \mu \mathrm{l}$ SE buffer $(0.75 \mathrm{M} \mathrm{NaCl}, 25 \mathrm{~mm}$ EDTA), the cells were lysed and the proteins were denatured by addition of $15 \mu \mathrm{l}$ of Proteinase K $(5 \mathrm{mg} / \mathrm{ml}$, Roche Molecular Diagnostics, Mannheim, Germany) and $145 \mu \mathrm{l}$ of $20 \%$ SDS with incubation for $2 \mathrm{~h}$ at $37^{\circ} \mathrm{C}$. The DNA was then extracted using an ethanol precipitation. DNA concentration was determined spectrophotometrically and the samples were diluted to a final concentration of $25 \mathrm{ng} / \mu \mathrm{l}$ of DNA.

\section{Candidate region selection and determination}

Chromosome $14 \mathrm{q} 32$ was treated as a candidate region because the human orthologue of the gene Lrd was originally reported to be located in this region ${ }^{14}$ although it is now known that this gene is located at chromosome 7p22 (M Brueckner, personal communication). Markers D14S272, D14S260, D14S542 and D14S1007, located on chromosome $14 \mathrm{q} 32$ near the Ig constant heavy-chain region, were employed to analyse this region.

The candidate regions for the other dyneins were determined through a combination of the information present in different databases and the study by Vaughan et al. ${ }^{9}$ The candidate region for Dnahc1 (Dynein Axonemal Heavy-Chain 1) is located on murine chromosome 14 , and the human syntenic region is chromosome 3p21-14, which contains markers D3S3647 and D3S1300. Human chromosome $6 \mathrm{p} 21$ is the candidate region for DNAHC6 and DNAHC8, which are localised on mouse chromosome 6 and 17 respectively. Dnahc13 is localised on mouse chromosome 12 with the human syntenic region being at $14 \mathrm{q} 32 .{ }^{9}$ The same markers were analysed for this region as for the candidate region of Lrd. A cytoplasmic dynein heavy-chain (Dnchc1) has the same localisation in mouse and human as Dnahc13. Dnahc2 has been localised to mouse chromosome 11 and human chromosome 17p13, which contains markers D17S786 and D17S1828.

The human NODAL gene is located in a $4 \mathrm{cM}$ interval on chromosome 10 between markers D10S210 and D10S537 (Genemap 99 http://www.ncbi.nlm.nih.gov/genemap/). To analyse this region, markers D10S1670, which was located less than $1 \mathrm{cM}$ away from D10S210, and D10S537 were chosen.

\section{Radiation hybrid mapping}

The Genebridge4 Radiation hybrid mapping panel was used to determine the localisation of the $L R D$ gene. Primers RG252K1 (TCACATGAACTGTAATGTTTG) and RG252K2 (AATAACACATCCTGGTCATAC) were employed in the PCR on the panel. 


\section{Microsatellite analysis}

Fragments were amplified with different annealing temperatures between $52^{\circ} \mathrm{C}$ and $58^{\circ} \mathrm{C}$ depending on the microsatellite, on a Geneamp 9700 PCR system (Perkin Elmer, Foster City, USA). PCR conditions included an initial 2 min denaturation step at $94^{\circ} \mathrm{C}$ followed by 33 cycles of denaturation at $94^{\circ} \mathrm{C}$ for $45 \mathrm{~s}$, annealing for $45 \mathrm{~s}$ at the specified temperature and extension for $45 \mathrm{~s}$ at $72^{\circ} \mathrm{C}$. An additional 2 min extension step at $72^{\circ} \mathrm{C}$ was used as the final step in every PCR reaction. PCR reactions were performed in a final volume of $20 \mu \mathrm{l}$ using $25 \mathrm{ng}$ of DNA, 4 pmol of each dNTP, $100 \mathrm{ng}$ of primer, a standard reaction buffer and a final $\mathrm{MgCl}_{2}$ concentration between 1 and $2 \mathrm{~mm}$ (depending on the microsatellite).

The markers for chromosome 14 were fluorescently endlabelled and separation of the PCR products was done on a Corbett fragment analyser (Corbett Research, Sydney, Australia). Other microsatellites were radioactively endlabelled with $\gamma\left[{ }^{33} \mathrm{P}\right]$-dATP and the fragments were separated on a $6.5 \%$ denaturing acrylamide gel.

\section{Data analysis}

Linkage analysis and simulation studies were done using the programs of the Linkage package. ${ }^{15}$ Genehunter ${ }^{16}$ was used for the non-parametric analysis on the nuclear families. Two genetic models were used in the parametric data analysis. The model by $\mathrm{Klar}^{7}$ was coded as autosomal recessive with 50\% penetrance to account for the $50 \%$ chance of becoming right-handed with an $\mathrm{r} / \mathrm{r}$ genotype. Gene frequencies were set at 58 and $42 \%$ for the $\mathrm{R}$ and $\mathrm{r}$ frequencies respectively, as postulated by Klar. ${ }^{7}$ The model of McManus $^{6}$ was coded as autosomal dominant with $50 \%$ penetrance for the $\mathrm{C} / \mathrm{C}$ genotype and $25 \%$ penetrance for the $\mathrm{D} / \mathrm{C}$ genotype. McManus proposed the gene frequency for the $\mathrm{C}$ and $\mathrm{D}$ allele to be 15.5 and $84.5 \%$ respectively in the general population. ${ }^{6}$ We used these frequencies in our linkage calculations using the model of McManus.

\section{Results}

\section{Candidate regions}

Left-handedness can be considered as a feature of left-right asymmetry. Hence genes involved in the establishment of left-right asymmetry can be considered as candidate genes. ${ }^{11}$ Because the murine gene Lrd, an axonemal heavy-chain dynein, is involved in the development of left-right asymmetry, ${ }^{8}$ the region of the human LRD gene and other axonemal dyneins were treated as candidate regions. An overview of the analysed axonemal dynein heavy-chain candidate regions is given in Table 1 (see Materials and methods for a more detailed description of the determination of the candidate regions and the chosen microsatellites).

\section{Localisation of the human LRD gene}

To determine the localisation of the human orthologue of $L r d$, the sequence of the Lrd gene was used in a Blast search which showed that BAC RG252K19 contains the human orthologue. Radiation hybrid mapping using the Genebridge 4 panel with two primers from BAC RG252K19 localised RG252K19 and thus $L R D$, telomeric to marker D7S531 on chromosome 7p22 (see Table 1 for the microsatellites used to analyse this region.)

\section{Candidate gene analysis using the extended pedigree}

Allele sharing results in the extended pedigree Manual allele sharing analysis was employed to study the different candidate regions in the extended pedigree. The model of Klar stipulates that all left-handed individuals must be $\mathrm{r} / \mathrm{r}$ homozygous. Therefore the left-handed parent of a righthanded child can pass either of the $\mathrm{r}$ alleles to any child, whether the child is right- or left-handed. The feature of fluctuating asymmetry in the genetic model means that right-handed individuals can also have the $\mathrm{r} / \mathrm{r}$ genotype. Right-handed parents who are $\mathrm{r} / \mathrm{r}$ homozygous would also pass on either of the $r$ alleles to their left-handed children.

Due to the high gene frequency of the $r$ allele in the general population, an r-allele introduced into the pedigree by a non-founder may not have the same surrounding haplotype as the r-allele of the founders. This can result in two different marker haplotypes in $\mathrm{r} / \mathrm{r}$ homozygous parents. ${ }^{11}$ Consequently, left-handed children of these $\mathrm{r} / \mathrm{r}$ right-handed parents could still be linked to the same locus without having inherited the same marker haplotype. ${ }^{11}$ These characteristics have to be taken into account when performing segregation analysis and can be used to measure a degree of linkage.

Table 1 Overview of the different candidate regions

\begin{tabular}{lcccc}
\hline Localisation & Gene & Location & Size & Microsatellites \\
\hline Chromosome 3 & DNAHC1 & $3 \mathrm{p} 21$ & $10 \mathrm{cM}$ & D3S3647, D3S1300 \\
Chromosome 6 & DNAHC6/DNAHC8 & $6 \mathrm{p} 21$ & $7 \mathrm{cM}$ & D6S464, D6S1616 \\
Chromosome 7 & LRD & $7 \mathrm{p} 22$ & $29 \mathrm{cM}$ & D7S2477, D7S1532, D7S531, D7S2508, D7S3047 \\
Chromosome 10 & NODAL & $10 \mathrm{p} 15$ & D10S1670, D10S537 cM & D14S272, D14S260, D14S542, D14S1007 \\
Chromosome 14 & DNAHC13 & $14 \mathrm{q} 32$ & D17S786, D17S1828 \\
Chromosome 17 & DNAHC2 & $17 \mathrm{p} 13$ & $10 \mathrm{cM}$ & D \\
\hline
\end{tabular}

The size of the different regions is given in centiMorgans (cM). 
Our analysis compared the observed segregation pattern to the ideal segregation pattern one would expect under complete co-segregation between left-handedness and the linked marker. Practically, this implied assessing the amount of observed allele sharing between left-handed individuals. If left-handed individuals do not show allele sharing, it will be necessary to invoke various $\mathrm{r} / \mathrm{r}$ genotypes in right-handed individuals to make the segregation pattern correspond to a linked segregation pattern. Therefore the higher the number of $\mathrm{r} / \mathrm{r}$ haplotypes that have to be assigned to right-handed individuals, reflecting the lower amount of allele sharing in left-handed subjects, the less likely a region will be linked. On the other hand, if no assumptions have to be made this will indicate complete allele sharing in left-handed individuals and the agreement with the segregation pattern of a linked marker. It is then feasible to assign degrees of possible linkage to the analysed candidate regions.

The NODAL candidate region on chromosome 10 shows allele sharing on the left side of the pedigree with lefthanded individuals sharing haplotype $4: 2$ at markers D10S1670 and D10S210 (Figure 1). Complete segregation of this haplotype can be observed in this side of the pedigree, suggesting co-segregation of left-handedness with these markers. On the right side of the family, the situation is more complex as only individual IV:26 has a haplotype (haplotype 6:8) from one of the founders (Figure 1). Based on the haplotypes of individuals IV:26, IV:27, IV:28 and IV:37, we have to invoke a $r / r$ genotype in individual II : 6 to make the data fit with linkage. Hence, it can be concluded that the results on the left side of the pedigree are promising but need confirmation by linkage analysis and candidate gene analysis in nuclear families.

For the DNAHC13 candidate region on chromosome 14, haplotype $4: 4: 3: 1$, at markers D14S272, D14S260, D14S542 and D14S1007, seems to segregate through the left side of the pedigree (Figure 2). However, individual $\mathrm{V}: 11$ does not have the $4: 4: 3: 1$ haplotype but this is possible as both parents are left-handed. Although individuals IV: 9 and IV: 10 share the $4: 4: 3: 1$ haplotype, it is possible that this is by chance if the maternal haplotype is homozygous $4: 4: 3: 1$. On the right side the $4: 4: 3: 1$ haplotype is not shared between the left-handed individuals. However, it can be concluded from the haplotypes of subjects IV:37, $\mathrm{V}: 14$ and $\mathrm{V}: 15$ who share haplotype $1: 3: 2: 4$, that lefthanded individual II $: 5$ has passed on the other haplotype. To explain the haplotypes of subjects IV:26, IV:27 and IV: 28 we have to invoke a $\mathrm{r} / \mathrm{r}$ genotype in subject III : 14 (Figure 2). In conclusion, the small number of right-handed people that need to have an $\mathrm{r} / \mathrm{r}$ genotype invoked suggests possible linkage to chromosome 14 .

No allele sharing was observed in the extended pedigree for the other candidate regions (data not shown). Thus it can be concluded that the NODAL and DNAHC13 regions on chromosomes 10 and 14 require further investigation.

\section{Linkage analysis using the extended pedigree}

Linkage analysis with Klar's model was used to analyse the different candidate regions in the extended pedigree. The highest LOD scores were obtained for the NODAL candidate region with a LOD score of 1.62 at $\theta=0.0$ for marker D10S1670 (Table 2). These results support the segregation analysis results as the NODAL region showed the highest degree of allele sharing. However, no significant negative LOD scores were obtained for the other regions despite the observed lack of allele sharing (Table 2), thereby illustrating the lack of power of the extended pedigree for linkage analysis.

To assess the significance of the linkage analysis results, we performed a simulation analysis of the extended pedigree using the program SLINK. ${ }^{17,18}$ A maximum obtainable LOD score of 1.69 was obtained. This shows that

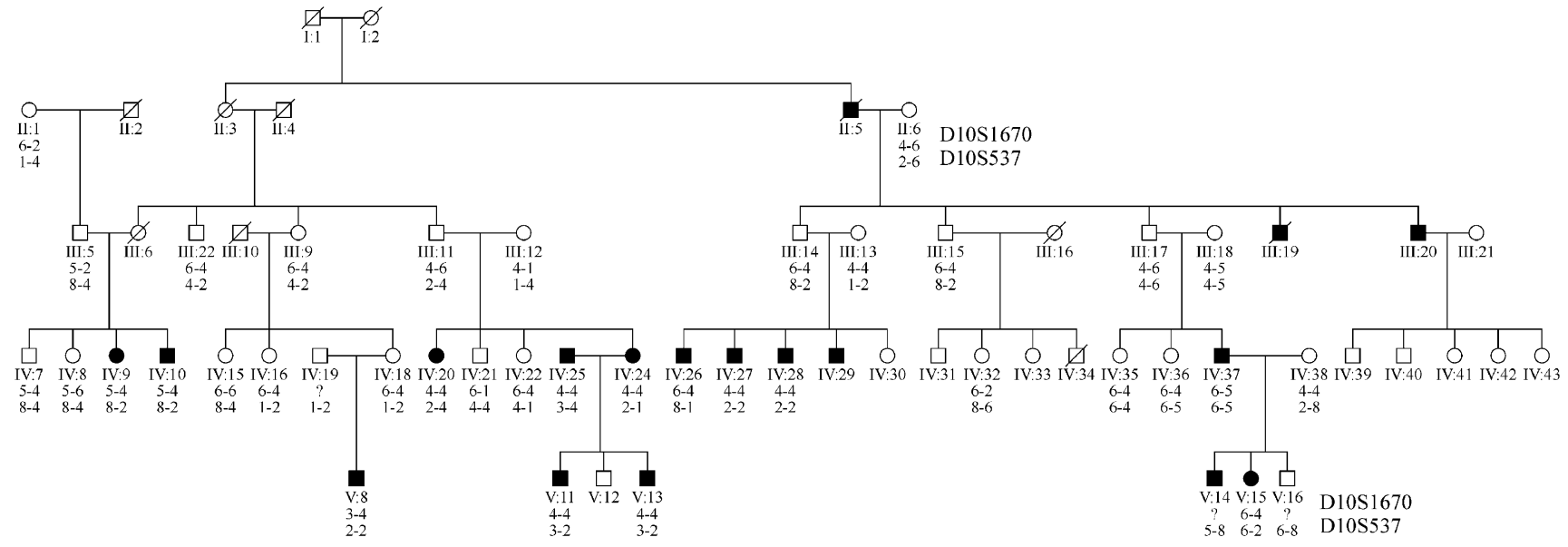

Figure 1 Microsatellite analysis of the chromosome 10 candidate region in the extended pedigree. Left-handed individuals are represented by filled symbols. 


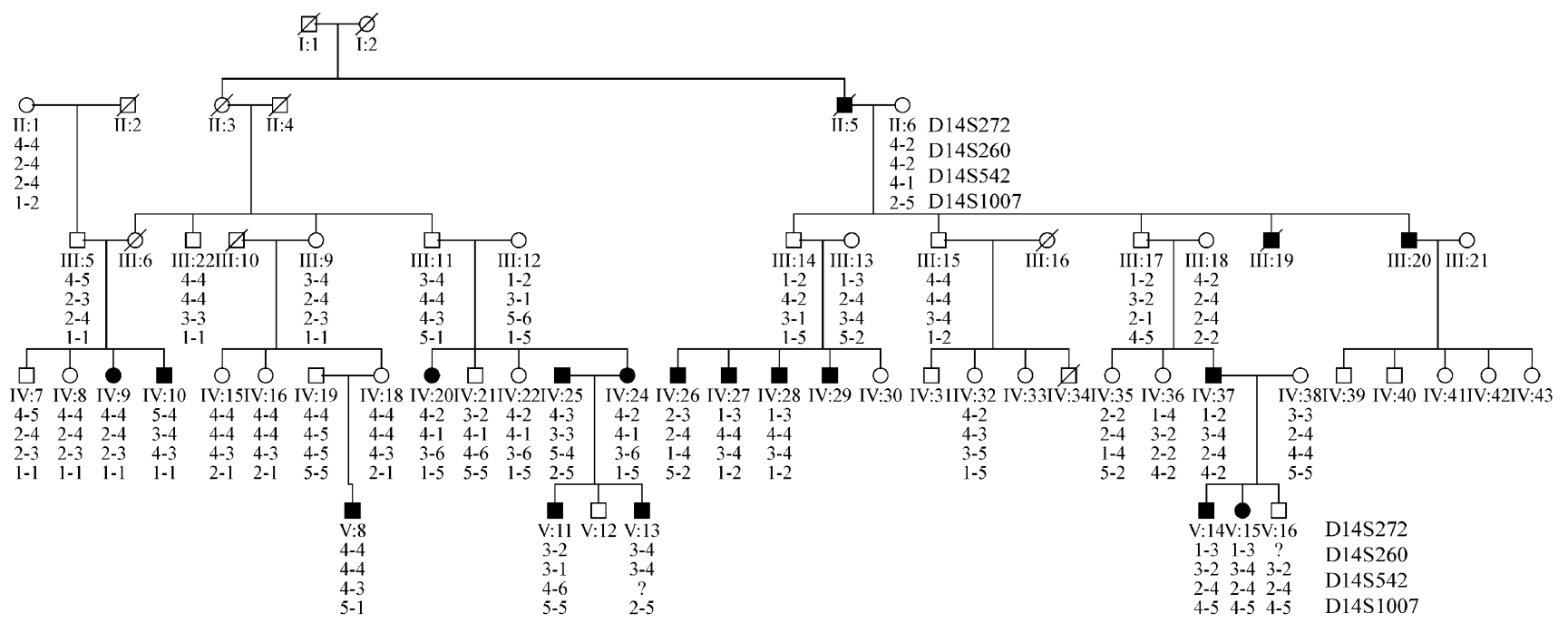

Figure 2 Microsatellite analysis of the chromosome 14 candidate region in the extended pedigree. Left-handed individuals are represented by filled symbols.

Table 2 Allele sharing results of the candidate regions in the nuclear families

\begin{tabular}{lcccc}
\hline & $\begin{array}{c}\text { Allele } \\
\text { sharing }\end{array}$ & $\begin{array}{c}\text { No allele } \\
\text { sharing }\end{array}$ & $\begin{array}{c}\text { Number } \\
\text { of family }\end{array}$ & $\begin{array}{c}\% \\
\text { sharing }\end{array}$ \\
\hline D3S3647 & 7 & 8 & 17 & 41 \\
D3S1300 & 5 & 15 & 20 & 25 \\
D6S464 & 8 & 13 & 21 & 38 \\
D6S1616 & 6 & 19 & 25 & 24 \\
D7S2477 & 8 & 14 & 22 & 36.40 \\
D751532 & 9 & 15 & 24 & 375 \\
D7S531 & 7 & 11 & 18 & 38.90 \\
D7S2508 & 6 & 18 & 24 & 25 \\
D2S3048 & 6 & 18 & 24 & 25 \\
D10S1670 & 6 & 12 & 18 & 33.30 \\
D10S537 & 6 & 14 & 20 & 30 \\
D14S272 & 6 & 10 & 16 & 37.50 \\
D14S260 & 7 & 18 & 25 & 28 \\
D14S542 & 7 & 18 & 25 & 28 \\
D14S1007 & 7 & 16 & 23 & 30.40 \\
D1751828 & 8 & 16 & 24 & 33.30 \\
D17S786 & 3 & 19 & 22 & 13.60 \\
\hline
\end{tabular}

The number of families for which data could be collected is given.

the obtained LOD score of 1.62 for marker D10S1670 warrants further investigation of this region using nuclear families. Hence, 27 nuclear families consisting of righthanded parents with left-handed children were used to further analyse the different candidate regions.

\section{Candidate gene analysis using nuclear families} Allele sharing analysis on nuclear families

An overview of the allele sharing analysis of the different candidate regions in the nuclear families is given in Table 3 . Based on the model of Klar, one can expect $75 \%$ allele sharing for a linked marker in the different nuclear families. ${ }^{11}$ The largest amount of allele sharing observed with these microsatellites is $41 \%$ for marker D3S3647. Based on these results it is unlikely that any of these markers are linked to a locus for handedness. However, to complement this analysis, parametric and non-parametric linkage analysis was performed on the nuclear families.

Linkage analysis using Klar's model

The results of the linkage analysis are given in Table $4 \mathrm{a}$. At $\theta=0.1$, markers D3S1300 and D6S1616 gave LOD scores of -2.93 and -2.37 respectively thereby excluding the chromosome 3 (DNAHC1) and chromosome 6 (DNAHC6 and DNAHC8) candidate regions. Linkage analysis for chromosome 7 resulted in the exclusion of a proportion of the candidate region. However, because the LOD values obtained with D7S2477 exclude the region between D7S2477 and D7S531, as a LOD value of -2.44 at $\theta=0.05$ was obtained, and $L R D$ is located in the $3 \mathrm{~cm}$ interval between D7S531 and D7S2477, the LRD candidate region can be excluded. The NODAL candidate region can be excluded by combining the regions excluded by the LOD scores of markers D10S1670 and D10S537. Two-point analysis of the DNAHC13 candidate region on chromosome 17 resulted in the complete exclusion of the interval as marker D17S786 gave a LOD score of -2.86 at $\theta=0.1$. It can therefore be concluded that all candidate regions can be excluded from harbouring a gene for human handedness with the model of Klar.

Linkage analysis using the model of McManus

The calculations using the model of McManus resulted in non-significant LOD scores for all markers analysed on 
Table 3 LOD score table of the different candidate regions analysed with the extended pedigree

\begin{tabular}{|c|c|c|c|c|c|c|c|}
\hline \multicolumn{8}{|c|}{ Klar } \\
\hline D3S3647 & -0.12 & -0.06 & 0.08 & 0.15 & 0.18 & 0.13 & 0.07 \\
\hline D3S1300 & -0.12 & -0.05 & 0.16 & 0.3 & 0.36 & 0.26 & 0.11 \\
\hline D6S1616 & -0.65 & -0.58 & -0.38 & -0.23 & -0.09 & -0.03 & -0.01 \\
\hline D7S2477 & -0.09 & -0.05 & 0.04 & 0.09 & 0.08 & 0.05 & 0.02 \\
\hline D7S1532 & -0.74 & -0.65 & -0.38 & -0.18 & 0.02 & 0.06 & 0.04 \\
\hline D7S3048 & -0.21 & -0.17 & -0.06 & 0.01 & 0.04 & 0.03 & 0.01 \\
\hline D10S1670 & 1.62 & 1.57 & 1.38 & 1.16 & 0.74 & 0.38 & 0.11 \\
\hline D10S537 & -0.43 & -0.35 & -0.14 & 0 & 0.07 & 0.01 & -0.05 \\
\hline D14S272 & 0.27 & 0.3 & 0.38 & 0.39 & 0.31 & 0.18 & 0.08 \\
\hline D14S260 & -0.6 & -0.53 & -0.31 & -0.15 & 0 & 0.04 & 0.04 \\
\hline D14S542 & -0.19 & -0.09 & 0.12 & 0.24 & 0.27 & 0.2 & 0.1 \\
\hline D14S1007 & 0.62 & 0.6 & 0.53 & 0.45 & 0.3 & 0.18 & 0.07 \\
\hline
\end{tabular}

Table 4 Linkage analysis results of the different candidate regions in the nuclear families using the models of Klar (a) and McManus (b)

\begin{tabular}{|c|c|c|c|c|c|c|c|}
\hline \multicolumn{8}{|l|}{ a Klar } \\
\hline theta & 0 & 0.01 & 0.05 & 0.1 & 0.2 & 0.3 & 0.4 \\
\hline D3S1300 & -6.7 & -6.15 & -4.42 & -2.93 & -1.22 & -0.42 & -0.09 \\
\hline D6S464 & -2.86 & -2.53 & -1.60 & -0.93 & -0.27 & -0.05 & 0.00 \\
\hline D7S2477 & -4.07 & -3.66 & -2.44 & -1.45 & -0.45 & -0.09 & -0.01 \\
\hline D7S1532 & -2.71 & -2.36 & -1.31 & -0.53 & 0.11 & 0.18 & 0.06 \\
\hline D7S531 & -2.50 & -2.21 & -1.32 & -0.66 & -0.07 & 0.05 & 0.02 \\
\hline D10S1670 & -2.97 & -2.71 & -1.91 & -1.26 & -0.53 & -0.19 & -0.04 \\
\hline D10S537 & -2.48 & -2.15 & -1.16 & -0.42 & 0.16 & 0.19 & 0.07 \\
\hline D14S272 & -3.18 & -2.92 & -2.09 & -1.40 & -0.61 & -0.22 & -0.05 \\
\hline D14S260 & -5.06 & -4.59 & -3.10 & -1.89 & -0.62 & -0.15 & -0.02 \\
\hline D14S542 & -3.92 & -3.47 & -2.18 & -1.21 & -0.29 & -0.01 & 0.01 \\
\hline D14S1007 & -4.58 & -4.18 & -2.96 & -1.92 & -0.77 & -0.25 & -0.05 \\
\hline D17S1828 & -4.11 & -3.66 & -2.33 & -1.28 & -0.27 & 0.02 & 0.03 \\
\hline \multicolumn{8}{|l|}{ b McManus } \\
\hline D3S1300 & -2.3 & -2.13 & -1.59 & -1.11 & -0.51 & -0.2 & -0.04 \\
\hline D6S464 & -0.98 & -0.88 & -0.59 & -0.35 & -0.10 & -0.02 & 0.00 \\
\hline D6S1616 & -2.12 & -1.96 & -1.43 & -0.98 & -0.44 & -0.17 & -0.04 \\
\hline D17S1828 & -1.00 & -0.87 & -0.50 & -0.22 & 0.02 & 0.05 & 0.02 \\
\hline D17S786 & -2.54 & -2.33 & -1.68 & -1.13 & -0.49 & -0.18 & -0.04 \\
\hline
\end{tabular}

chromosomes 7, 10 and 14 (data not shown). Analysis of the regions on chromosomes 3, 6 and 17 resulted in significant negative LOD scores for one of the analysed markers (Table 4b).

\section{Non-parametric linkage analysis}

Non-parametric analysis failed to reach significant values for any of the analysed markers (data not shown). The highest NPL score was obtained for marker D10S537 which gave a NPL score of 1.13 with a pointwise $P$-value of 0.12 . However marker D10S1670, which gave the highest LOD score in the extended pedigree, gave a negative NPL score of -0.27 with a $P$-value of 0.58 . It can therefore be concluded that the NPL analysis confirms the manual allele sharing analysis. 
Based on the linkage analysis results in the nuclear families, all candidate regions can be excluded from harbouring a gene with the models of Klar and McManus which is supported by the data of the non-parametric analysis.

\section{Discussion}

Left-handedness has been studied intensively, although only a very small number of molecular projects have been undertaken and the genetic model of human handedness still remains to be elucidated. Because the inheritance pattern of handedness in humans does not follow a classic Mendelian inheritance pattern, it is often considered as a complex trait. ${ }^{19}$ However, the proposed genetic models such as those of $\mathrm{Klar}^{7}$ and McManus ${ }^{6}$ consider handedness as a single gene trait with a non-Mendelian inheritance pattern.

In order to test the feasibility of mapping genes for human handedness we collected 27 nuclear families consisting of right-handed parents and at least two lefthanded children, and an extended pedigree to analyse different candidate regions. Left-handedness was modelled as a single gene disorder with a non-Mendelian inheritance pattern and candidate gene analysis was performed using the models of Klar and McManus. The genetic right-shift model of Annett, ${ }^{20}$ in which a right shift increases the chance of humans being right-handed, was not used separately in the analysis as her model is to a certain degree quite similar to the model of McManus. ${ }^{6,20,21}$ However underlying differences exist between the two models in their description of handedness. ${ }^{21}$ Annett based her model on the unimodal distribution of skill differences in handedness $^{20}$ rather than the bimodal distributed direction of handedness (used by Klar and McManus), which indicates what hand is used for unimanual tasks. It is the direction of handedness that is more genetically determined than the difference in skill. ${ }^{6}$

Manual segregation analysis of the extended pedigree identified allele sharing in the NODAL and DNAHC13 candidate regions on chromosomes 10 and 14 . LOD score analysis resulted in non-significant LOD scores for all candidate regions with a maximum LOD score of 1.62 for marker D10S1670 thereby supporting the results of the segregation analysis of this region. However, similar LOD scores were obtained for the regions on chromosome 14 and 17, despite a marked difference in degree of allele sharing. These results indicate the problematic nature of manual segregation analysis and lack of sufficient power in the pedigree for linkage analysis using Klar's model.

The problems with the segregation analysis are caused by the characteristics of Klar's model. The fluctuating asymmetry component of the model creates the possibility that right-handed individuals have the same genotype at the trait locus as left-handed individuals. ${ }^{7}$ Left-handed children from these right-handed individuals do not have to share the marker haplotype as the right-handed individuals could have passed on either allele to their children. ${ }^{11}$ In addition, due to the high gene frequency of the $r$ allele, it is very likely that the $r$ allele will be introduced into the family by a founder as well as non-founders. Therefore it is possible to invoke the presence of a second handedness determining haplotype (introduced by a non-founder) to explain the lack of allele sharing of the ancestral haplotype between left-handed individuals. Based on this, the high gene frequency and the fluctuating asymmetry in the model make it possible to make any data fit a scenario of linkage. This makes it impossible to exclude any candidate region and the segregation analysis merely becomes a tool to classify the degree of allele sharing or linkage.

Subsequent allele sharing analysis of the candidate regions in 27 nuclear families indicated that the highest amount of allele sharing was $41 \%$ for D3S3647. Given the fact that $75 \%$ of families linked to a region will show complete allele sharing, ${ }^{11}$ these results indicate the possible exclusion of the analysed regions. Additional LOD score analysis with the model of Klar resulted in the complete exclusion of all regions thereby confirming the allele sharing results. Non-parametric analysis and analysis with the model of McManus resulted in non-significant results except for markers D3S1300, D6S1616, D7S3048 and D17S796 which gave significant negative results with the model of McManus.

These results indicate that it is unlikely that a recessive gene is linked to any of the markers tested in these families. In addition, assuming that left-handedness is monogenic, these results show that the positive result in the extended family of chromosomes 10 is a false positive result. However, it should be noted that as we have treated human handedness as a monogenic trait with a non-Mendelian inheritance pattern, the exclusion only applies to genes with the specified genetic model. Results from the nonparametric analysis support the exclusion of the analysed candidate regions.

Although Corballis ${ }^{22}$ has argued against it, several reports have suggested that a locus for handedness is located on the X-chromosome ${ }^{23,24}$ which can act through X-inactivation in females. ${ }^{25}$ Association analysis on 180 pairs of lefthanded brothers resulted in a suggestive LOD score of 2.8 with marker DXS990 26 if the data were analysed for a difference in variance of left-hand skill. However it has been argued that it is the direction of handedness rather than the skill difference that is genetically determined whilst the degree and thus skill is more environmentally determined. ${ }^{6}$ In addition earlier reports ${ }^{22,27}$ at the time of study did not specify a specific locus which made the $\mathrm{X}$ chromosome unsuitable for a candidate gene analysis. However very recently Crow $^{28}$ has suggested Protocadherin$\mathrm{XY}$ as the likely candidate.

The enormous progress that has been made in the elucidation of the left-right asymmetry pathway has resulted in 
a plethora of candidate genes. Given the substantial number of candidate genes a genome scan on handedness, instead of candidate gene analysis, is recommended. A genome scan would also reveal the absence or presence of a locus with a major effect, thereby providing more information regarding the genetics of human handedness.

\section{Acknowledgements}

The Murdoch Childrens Research Institute is a block funded research institute by the National Health and Medical Research Council of Australia. We wish to acknowledge the support and co-operation of all the families who took part in this study. The authors would like to thank Dr Les Sheffield and Dr Mark McCarthy for helpful discussion.

\section{References}

1 Gilbert AN, Wysocki CJ: Hand preference and age in the United States. Neuropsychologia 1992; 30: 601-608.

2 Olsson H, Ingvar C: Left handedness is uncommon in breast cancer patients. Eur J Cancer 1991; 27: 1694-1695.

3 Coren S, Halpern DF: Left-handedness: a marker for decreased survival fitness. Psychol Bull 1991; 109: 90-106.

4 Geschwind N, Galaburda AM: Cerebral lateralization. Cambridge: MIT Press, 1987, pp 67-79.

5 Knecht S, Drager B, Deppe M et al: Handedness and hemispheric language dominance in healthy humans. Brain 2000; 123: 2512 2518 .

6 McManus IC: Handedness, language dominance and aphasia: a genetic model. Psychol. Med 1985; Monograph Suppl No 8.

7 Klar AJ: A single locus, RGHT, specifies preference for hand utilization in humans. Cold Spring Harb Symp Quant Biol 1996; 61: 59 65.

8 Supp DM, Witte DP, Potter SS, Brueckner M: Mutation of an axonemal dynein affects left-right asymmetry in inversus viscerum mice. Nature 1997; 389: 963 -966.

9 Vaughan KT, Mikami A, Paschal BM et al: Multiple mouse chromosomal loci for dynein-based motility. Genomics 1996; 36: $29-38$.

10 Levin M, Johnson RL, Stern CD, Kuehn M, Tabin C: A molecular pathway determining left-right asymmetry in chick embryogenesis. Cell 1995; 82: 803-814.
11 Van Agtmael T, Forrest SM, Williamson R: Genes for left-handedness: How to search for the needle in the haystack? Laterality 2001; 6: 149-164.

12 Oldfield RC: The assessment and analysis of handedness: the Edinburgh inventory. Neuropsychologia 1971; 9: 97-113.

13 Lench N, Stanier P, Williamson R: Simple non-invasive method to obtain DNA for gene analysis. Lancet 1988; 1: 1356-1358.

14 Brueckner M, D'Eustachio P, Horwich AL: Linkage mapping of a mouse gene, iv, that controls left-right asymmetry of the heart and viscera. Proc Natl Acad Sci USA 1989; 86: 5035-5038.

15 Lathrop GM, Lalouel JM, Julier C, Ott J: Strategies for multilocus linkage analysis in humans. Proc Natl Acad Sci USA 1984; 81 $3443-3446$.

16 Kruglyak L, Daly MJ, Reeve-Daly MP, Lander ES: Parametric and nonparametric linkage analysis: a unified multipoint approach Am J Hum Genet 1996; 58: 1347-1363.

17 Terwilliger JD, Speer M, Ott J: Chromosome-based method for rapid computer simulation in human genetic linkage analysis. Genet Epidemiol 1993; 10: 217-224.

18 Weeks DE, Ott J: G.M. L SLINK: a general simulation program for linkage analysis. Am J Hum Genet 1990; 47: A:204.

19 Lander ES, Schork NJ: Genetic dissection of complex traits. Science 1994; 265: 2037-2048.

20 Annett M: Left, right, hand and brain; the right shift theory. Hove, UK: Lawrence Erlbaum Associates Ltd, 1985, pp 283-301.

21 McManus IC: The inheritance of left-handedness. Biological asymmetry and handedness. Ciba Found Symp 1991; 162: 251 281.

22 Corballis MC: The genetics and evolution of handedness. Psych Rev 1997; 104: 714-727.

23 Corballis MC, Lee K, McManus IC, Crow TJ: Location of the handedness gene on the $\mathrm{X}$ and $\mathrm{Y}$ chromosomes. Am J Med Genet 1996; 67: 50-52.

24 Crow TJ: Schizophrenia as failure of hemispheric dominance for language. Trends Neurosci 1997; 20: 339-343.

25 Corballis MC: Is the handedness gene on the $\mathrm{X}$-chromosome? Psych Rev 2001; 108: $805-810$.

26 Laval SH, Dann JC, Butler RJ et al: Evidence for linkage to psychosis and cerebral asymmetry (relative hand skill) on the $\mathrm{X}$ chromosome. Am J Med Genet 1998; 81: 420-427.

27 McKeever WF: A new family handedness sample with findings consistent with X-linked transmission. Br J Psychol 2000; 91 : 21-39.

28 Crow TJ: ProtocadherinXY: A candidate gene for cerebral asymmetry and language; in Wray A (ed): The transition to language. Oxford: Oxford University Press, 2002, vol 1, pp 93-112. 